\title{
Accuracy requirements to test the applicability of the random cascade model to supersonic turbulence
}

\author{
Doris Folini and Rolf Walder

\begin{abstract}
École Normale Supérieure, Lyon, CRAL, UMR CNRS 5574, Université de Lyon, 69342 Lyon, France
e-mail: doris.folini@ens-lyon.fr
\end{abstract}

Received 9 August 2015 / Accepted 21 January 2016

\begin{abstract}
A model, which is widely used for inertial rang statistics of supersonic turbulence in the context of molecular clouds and star formation, expresses (measurable) relative scaling exponents $Z_{p}$ of two-point velocity statistics as a function of two parameters, $\beta$ and $\Delta$. The model relates them to the dimension $D$ of the most dissipative structures, $D=3-\Delta /(1-\beta)$. While this description has proved most successful for incompressible turbulence $(\beta=\Delta=2 / 3$, and $D=1)$, its applicability in the highly compressible regime remains debated. For this regime, theoretical arguments suggest $D=2$ and $\Delta=2 / 3$, or $\Delta=1$. Best estimates based on 3D periodic box simulations of supersonic isothermal turbulence yield $\Delta=0.71$ and $D=1.9$, with uncertainty ranges of $\Delta \in[0.67,0.78]$ and $D \in[2.04,1.60]$. With these $5-10 \%$ uncertainty ranges just marginally including the theoretical values of $\Delta=2 / 3$ and $D=2$, doubts remain whether the model indeed applies and, if it applies, for what values of $\beta$ and $\Delta$. We use a Monte Carlo approach to mimic actual simulation data and examine what factors are most relevant for the fit quality. We estimate that $0.1 \%(0.05 \%)$ accurate $Z_{p}$, with $p=1, \ldots, 5$, should allow for $2 \%$ (1\%) accurate estimates of $\beta$ and $\Delta$ in the highly compressible regime, but not in the mildly compressible regime. We argue that simulation-based $Z_{p}$ with such accuracy are within reach of today's computer resources. If this kind of data does not allow for the expected high quality fit of $\beta$ and $\Delta$, then this may indicate the inapplicability of the model for the simulation data. In fact, other models than the one we examine here have been suggested.
\end{abstract}

Key words. shock waves - hydrodynamics - ISM: kinematics and dynamics - gamma-ray burst: general - binaries: close - turbulence

\section{Introduction}

Supersonic turbulence is a key ingredient in various astrophysical contexts, from gamma ray bursts (Lazar et al. 2009; Narayan \& Kumar 2009) or stellar accretion (Walder et al. 2008; Hobbs et al. 2011) to molecular clouds and star formation (Chabrier \& Hennebelle 2011; Federrath \& Klessen 2012; Padoan et al. 2012; Kritsuk et al. 2013). A key question is whether this turbulence, like incompressible turbulence, is characterized by universal statistics. Results from 3D periodic box simulations of driven, isothermal, supersonic turbulence (Kritsuk et al. 2007a; Schmidt et al. 2008; Pan et al. 2009) are indeed consistent with the highly compressible variant (Boldyrev 2002) of the hierarchical structure model that was put forward by She \& Leveque (1994) for incompressible turbulence and that was further scrutinized by Dubrulle (1994) and She \& Waymire (1995). This model is correspondingly popular in astrophysics. It is employed, for example, in the interpretation of molecular cloud observations (Gustafsson et al. 2006; Hily-Blant et al. 2008) or to derive a theoretical expression for the density distribution in supersonic turbulence (Boldyrev et al. 2002), which enters theories of the stellar initial mass function (Hennebelle \& Chabrier 2008).

Nevertheless, some doubts remain whether the model really applies to simulation data of supersonic turbulence and, if so, with what parameter values. The best-fit model parameters that we are aware of (Pan et al. 2009) still come with a 5-10\% uncertainty range that is only marginally compatible with theoretically predicted parameter values (see below). Here we argue that today's computer resources should allow for 1-2\% accurate parameter fits in the highly compressible regime, thereby likely settling the issue. Our claim is based on a Monte Carlo approach to mimic actual simulation data.

The hierarchical structure model predicts the ratios $Z_{p}$ of (observable) structure function scaling exponents $\zeta_{p}, p=$ $1,2,3, \ldots$ etc., of a $3 \mathrm{D}$ velocity field $\boldsymbol{u}$ as

$Z_{p}=\frac{\zeta_{p}}{\zeta_{3}}=(1-\Delta) \frac{p}{3}+\frac{\Delta}{1-\beta}\left(1-\beta^{p / 3}\right)$.

Here, $D=3-C$ is the dimension of the most intermittent structure, $C=\Delta /(1-\beta)$ the associated co-dimension, $\beta \in[0,1]$ measures the intermittency of the energy cascade, and $\Delta \in[0,1]$ measures the divergent scale dependence of the most intermittent structures. The $\zeta_{p}$ are defined in the inertial range by

$S_{p}(r) \equiv\left\langle|\boldsymbol{u}(\boldsymbol{x}+\boldsymbol{r})-\boldsymbol{u}(\boldsymbol{x})|^{p}\right\rangle \propto r^{\zeta_{p}}$,

where $\langle\ldots\rangle$ denotes the average over all positions $\boldsymbol{x}$ within the sample and over all directed distances $r$. The $Z_{p}$ should be well defined over a larger range because of extended selfsimilarity (Benzi et al. 1993) and Eq. (1) should remain formally valid for generalized structure functions $\tilde{S}_{p}(r)$, computed from mass-weighted velocities $\boldsymbol{v} \equiv \rho^{1 / 3} \boldsymbol{u}$ (Kritsuk et al. 2007a,b).

Several special cases of the model that differ in their parameter values exist in the literature (see e.g. the review by She $\&$ Zhang 2009). The original model by She \& Leveque (1994) applies most successfully to incompressible turbulence with 1D vortex filaments as most dissipative structures $(D=1)$ and parameter values $\beta=\Delta=2 / 3$. For highly compressible turbulence, parameter values remain debated. Boldyrev (2002) argues that the most dissipative structures are $2 \mathrm{D}$ shocks, thus $D=2$, 
and chose to keep $\Delta=2 / 3$ and $\operatorname{set} \beta=1 / 3$. By contrast, Schmidt et al. (2008) argue that $\Delta=1$ (implying $\beta=0$ ) to be consistent with Burgers turbulence. A few studies used 3D simulation data, derived sets of $Z_{p}$, and attempted simultaneous fits of $\beta$ and $\Delta$ (Kritsuk et al. 2007b; Schmidt et al. 2008, 2009; Folini et al. 2014). The results are inconclusive in that fits of similar quality are obtained for widely different $\beta$ - $\Delta$-pairs. Also using 3D simulation data $\left(1024^{3}\right.$, Mach 6) but working with density-weighted moments of the dissipation rate, Pan et al. (2009) simultaneously fitted $\Delta$ and $D$ to their data. They find $\Delta \in[0.67,0.78]$ and $D \in[2.04,1.60]$, with a best estimate of $\Delta=0.71$ and $D=1.9$, thus $\beta=0.35$. The range for $\Delta$ is not compatible with the suggested $\Delta=1$ (see above), and also $\Delta=2 / 3$ lies only at the lower-most bound of the inferred range. Both $\Delta$ and $\beta$ may thus deviate from their incompressible values $(\beta=\Delta=2 / 3)$ as the Mach number increases, making simultaneous determination of $\beta$ and $\Delta$ a must.

The present study is motivated by this still inconclusive situation. We want to better understand what factors (accuracy/order of $Z_{p}$; mildly versus highly compressible turbulence) are most relevant for the fit quality and why widely different $\beta$ - $\Delta$-pairs yield fits of similar quality. We use this insight to formulate quantitative estimates of what is needed to obtain $1 \%$ accurate estimates of $\beta$ and $\Delta$. We present results in Sect. 2, discuss them in Sect. 3, and conclude in Sect. 4.

\section{Results}

We first show that $\beta$ and $\Delta$ can be uniquely determined from an associated (i.e. computed via Eq. (1)) pair $Z_{p_{1}}$ and $Z_{p_{2}}$. We then illustrate how uncertainties in $Z_{p}$ map onto the $\beta$ - $\Delta$-plane. Finally, we give estimates on how accurate the $Z_{p}$ have to be to achieve a desired accuracy of $\beta, \Delta$, and $C$.

\section{1. $\beta$ and $\Delta$ from exact $Z_{p}$}

Consider two values $Z_{p_{1}}$ and $Z_{p_{2}}$ that both fulfill Eq. (1) for the same values $(\beta, \Delta)$. In the following, we show that $(\beta, \Delta)$ can unambiguously (uniquely) be recovered from $Z_{p_{1}}$ and $Z_{p_{2}}$.

We start by rewriting Eq. (1), factoring out $\Delta$ :

$0=\Delta\left(\frac{3\left(1-\beta^{p / 3}\right)-p(1-\beta)}{3(1-\beta)}\right)+\left(\frac{p-3 Z_{p_{j}}}{3}\right), \quad j=1,2$.

Using $Z_{p_{1}}$, we can obtain an expression for $\Delta$,

$\Delta=\left(\frac{3 Z_{p_{1}}-p_{1}}{3}\right)\left(\frac{3(1-\beta)}{3\left(1-\beta^{p_{1} / 3}\right)-p_{1}(1-\beta)}\right)$.

By now writing Eq. (3) with $Z_{p}=Z_{p_{2}}$, using Eq. (4) to replace $\Delta$, do some re-ordering of terms, and abbreviating $p_{1} / 3 \equiv a$ and $p_{2} / 3 \equiv b$, we end up with the following equation for $\beta$ :

$0=\left(\frac{-\beta^{b}+b \beta+1-b}{-\beta^{a}+a \beta+1-a}\right)-\left(\frac{Z_{p_{2}}-b}{Z_{p_{1}}-a}\right) \equiv \frac{P_{\beta, b}}{P_{\beta, a}}-R_{a, b}$,

or

$R_{a, b} P_{\beta, a}=P_{\beta, b}$.

The polynomial $P_{\beta, x} \equiv-\beta^{x}+x \beta+1-x$, with $x>0$ and $\beta \in(0,1)$, is a monotonically decreasing (increasing) function for $x<1$ $(x>1)$, as can be seen by taking the derivative of $P_{\beta, x}$ with respect to $\beta$ and as illustrated in Fig. 1. Consequently, Eq. (6) has a unique solution, $\beta$, from which $\Delta$ can be recovered via

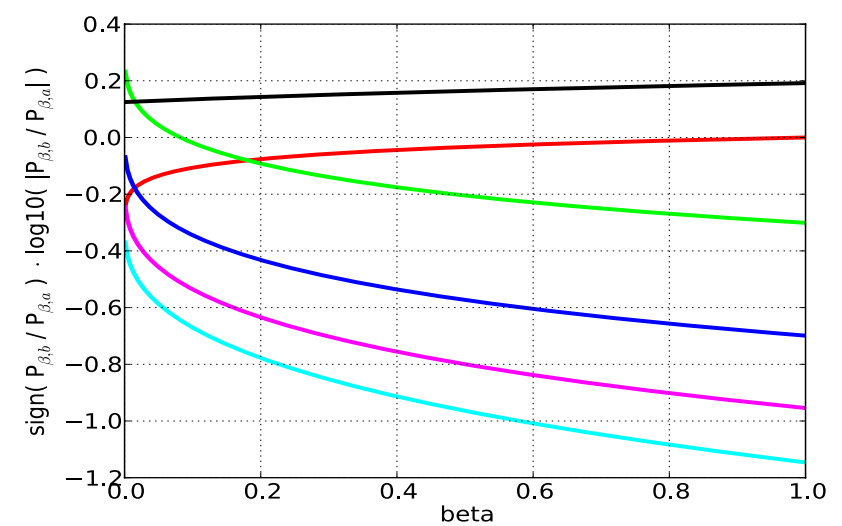

Fig. 1. Ratio of polynomials $P_{\beta, b} / P_{\beta, a}$, Eq. (5), (y-axis, shown as logarithm) for selected exponents $a$ and $b$ as function of $\beta$ (x-axis). Colors indicate $b / a=2$ (red), 4 (green), 5 (blue), 6 (magenta), 7 (cyan), all with $a=p_{1} / 3=1 / 3$, as well as $b / a=7 / 6$ (black) with $a=p_{1} / 3=6 / 3$.

Eq. (4). Thus Eq. (1) defines an exact one-to-one correspondence between pairs $\left(Z_{p_{1}}, Z_{p_{2}}\right)$ and $(\beta, \Delta)$.

Two more points deserve to be highlighted, with the help of Fig. 1. The ratio $P_{\beta, b} / P_{\beta, a}=R_{a, b}$ is shown as a function of $\beta$ for different $a$ and $b$ or, equivalently, $p_{1}$ and $p_{2}$. From the figure it can be taken that, first, largely different values of $p_{1}$ and $p_{2}$ are advantageous since they result in stronger stratification of $\beta$ with respect to $R_{a, b}=\left(Z_{p_{2}}-b\right) /\left(Z_{p_{1}}-a\right)$. The cyan curve in Fig. 1, which represents $p_{1} / p_{2}=1 / 7$, covers a wider range of values on the $\mathrm{y}$-axis than the black curve $\left(p_{1} / p_{2}=6 / 7\right)$. Secondly, the stratification is stronger for small $\beta$. Somewhat anticipating Sect. 2.2, we thus expect uncertainties in the $Z_{p}$ to be less important if $Z_{p}$ are available for largely different $p$ and if they are associated with (yet to be determined) small values of $\beta$.

\subsection{Uncertainty of $Z_{p}$ in the $\beta$ - $\Delta$-plane}

\subsubsection{Single $Z_{p}$}

From Eq. (4) it is clear that each $Z_{p}$ defines a curve in the $\beta$ - $\Delta$ plane. If $Z_{p}$ is derived from model data or observations, it will typically come with an uncertainty estimate, e.g. $\delta Z_{p} / Z_{p} \leq 5 \%$, with $\delta$ indicating the uncertainty. In the $\beta-\Delta$-plane, this uncertainty range translates into an area around the $Z_{p}$ curve. An illustration is given in Fig. 2. The following points may be made.

One value of $Z_{p}$ (a line of constant $Z_{p}$ in the $\beta$ - $\Delta$-plane) is compatible with a (large) range of $\beta$ and/or $\Delta$ that always includes $\Delta=1$ and $\beta=0$. The range tends to be smaller for $Z_{p}$ associated with small $\beta$ and large $\Delta$ (i.e. the lower right corner of $\beta$ - $\Delta$-plane). Uncertainties associated with $Z_{p}$ (5\% in Fig. 2 , white curves) augment the range, especially for $p=2$ and $p=4$, as well as for small $\Delta$ and large $\beta$ (top left corner of the plane). Also apparent from Fig. 2 (or from taking the derivative with respect to $\Delta$ of Eq. (1)): for fixed $\beta$ and $p<3(p>3), Z_{p}$ is a monotonically increasing (decreasing) function of $\Delta$. A similar statement holds for $Z_{p}$ as a function of $\beta$ for fixed $\Delta$.

In summary, we expect uncertainties in the $Z_{p}$ to be more of an issue if only low orders of $p$ (up to about 4 ) are available and/or if the (yet to be determined) $\beta$ is large.

\subsubsection{Multiple $Z_{p}$}

We now turn to multiple $Z_{p}$ and their associated uncertainty ranges $\delta Z_{p}$, and ask what area they define in the $\beta-\Delta$-plane. An 

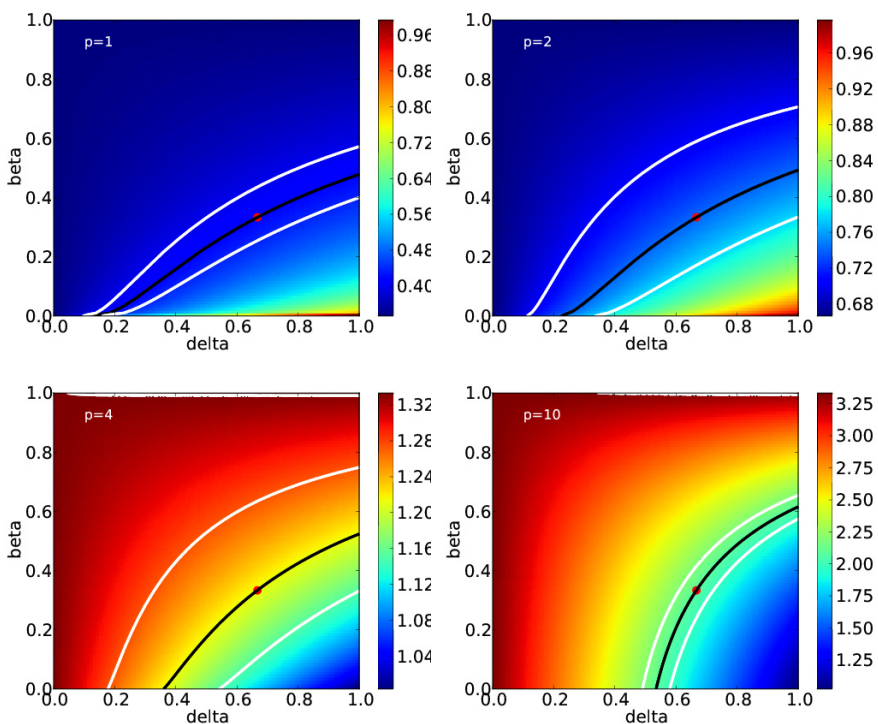

Fig. 2. $Z_{p}$ in the $\beta$ - $\Delta$-plane, role of $p$. Shown is $Z_{p}$ (color coded) for $p=1$ (top left), $p=2$ (top right), $p=4$ (bottom left) and $p=10$ (bottom right). For $\beta=1 / 3$ and $\Delta=2 / 3$ (red dot), the curve of constant $Z_{p}$ (black) is shown, as well as curves of $\pm 5 \%$ different $Z_{p}$ (white).
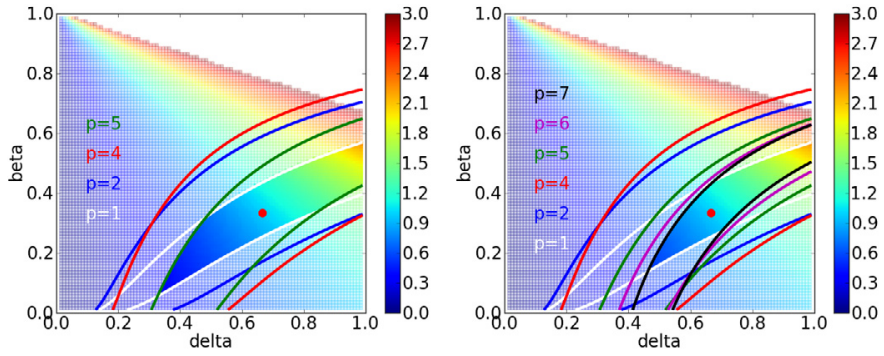

Fig. 3. Part of $\beta$ - $\Delta$-plane (color coded in co-dimension $C=\Delta /(1-\beta)$, $0<C<3$ ) within joint reach of (at most) $5 \%$ perturbed $Z_{p}$, starting from $Z_{p}$ for $\Delta=2 / 3$ and $\beta=1 / 3$ (red dot). Left panel: curves of $5 \%$ perturbed $Z_{p}$ values for $p=1$ (white), $p=2$ (blue), $p=4$ (red), and $p=5$ (green) and part of the $\beta$ - $\Delta$-plane enclosed by all of them. Right panel: same as left panel but also including $p=6$ (purple) and $p=7$ (black).

illustration is given in Fig. 3. Starting from one specific pair of $\beta=1 / 3$ and $\Delta=2 / 3$ and computing $Z_{p}$ for $p=1, \ldots, 5$ (left) or $p=1, \ldots, 7$ (right), we show pairs of $5 \%$ perturbed $Z_{p}$ curves, i.e. $1.05 \cdot Z_{p}$ and $0.95 \cdot Z_{p}$.

As can be seen, only a small fraction of the $\beta$ - $\Delta$-plane lies between all pairs of perturbed curves. Yet this area comprises a wide range of $(\beta, \Delta)$ values or co-dimensions. The $5 \%$ uncertainty in the $Z_{p}$ translates into a much larger uncertainty (in per cent) for $\beta$ and $\Delta$. Closer inspection reveals that the area is actually defined by only two sets of curves: those for $p=1$ and $p=5$ (left panel) or $p=7$ (right panel). The latter area is smaller, which indicates that higher order structure functions constrain the problem of finding $\beta$ and $\Delta$ from a set of $Z_{p}$ more strongly. Also apparent from Fig. 3 is the dominant role of the $p=1$ curve for narrowing down the composite area between all curves. All this is in line with the expectation (see Sect. 2.1) that $Z_{p}$ for largely different $p$ are advantageous for the determination of $\beta$ and $\Delta$.

The relevance of the overall location in the $\beta$ - $\Delta$-plane is illustrated in Fig. 4. Again, the area shown is contained within 5\% perturbed $Z_{p}$ curves for two additional $(\beta, \Delta)$ pairs. As can be seen, smaller values of $\beta$ (lower panels) result in smaller areas,
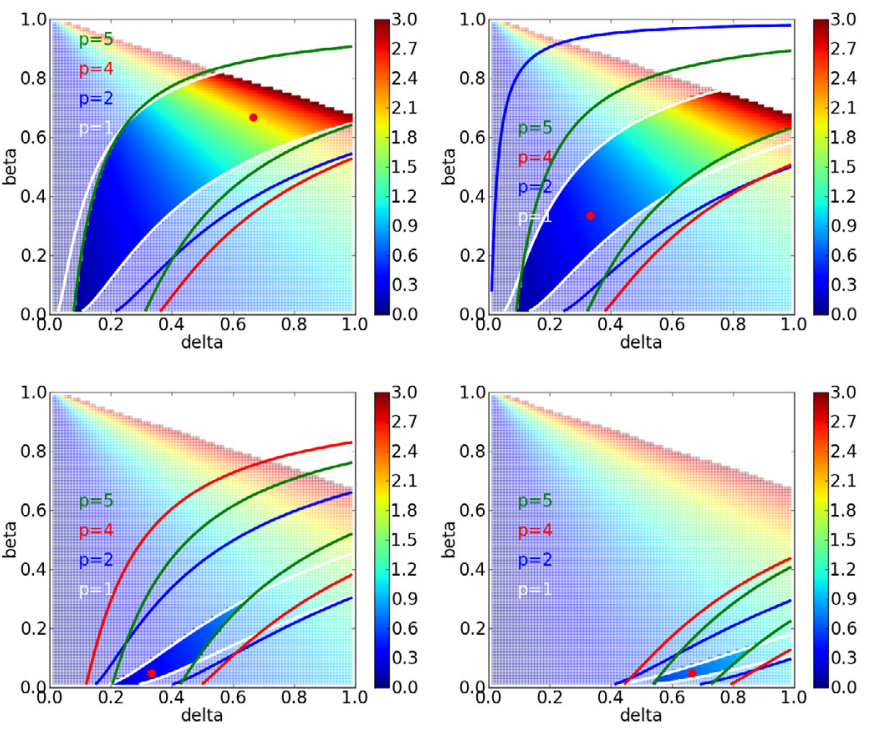

Fig. 4. Same as Fig. 3, left, but for $\beta=\Delta=2 / 3$ (top left), $\beta=\Delta=1 / 3$ (top right), $\beta=0.048$ and $\Delta=1 / 3$ (bottom left), as well as and $\beta=$ 0.048 and $\Delta=2 / 3$ (bottom right).

independent of $\Delta$. The crucial role of the $p=1$ (white) and $p=5$ (green) curves for confining the area persists. Table 1 gives a quantitative idea of the relevance of $\beta, \Delta, \delta Z_{p}$, and $p_{\max }$ for the uncertainty range $\pm \delta C$ of the co-dimensions $C$. A small $\delta C$ basically requires a small $\delta Z_{p}$, a large $p_{\max }$, a small $\beta$, and a large $\Delta$. The concrete numbers highlight the difficulty (or ill-posedness) of the problem. The situation is worse for larger $\beta$ (bottom rows in Table 1) and better for smaller values of $\beta$ (not shown).

We emphasize that the above considerations serve only as illustration. We looked at the area confined by a set of $Z_{p} \pm$ $\delta Z_{p}$ curves. We have not yet considered the problem of estimating best-fit $\beta_{\mathrm{f}}, \Delta_{\mathrm{f}}$, and thus $C_{\mathrm{f}}$ for a set of given $Z_{p}$. Such a best-fit solution may lie outside the area considered here.

In summary, very accurate $Z_{p}$ are needed to derive reliable best estimates for $\beta, \Delta$, and $C$, and smaller values of $\beta$ help.

\subsection{Best-fit $\beta_{f}$ and $\Delta_{f}$ from uncertain $Z_{p}$}

We now turn to our actual problem of interest: given a set of perturbed (uncertain) $\tilde{Z}_{p}=Z_{p}+\delta Z_{p}$, what are associated best-fit estimates for $\beta_{\mathrm{f}}$ and $\Delta_{\mathrm{f}}$ ? Different techniques exist to cope with this kind of question (e.g. Najm 2009; Le Maître \& Knio 2010). We use a simple Monte Carlo approach.

We start with a pair $(\beta, \Delta)$ and a maximum order $p_{\max }$, then use Eq. (1) to obtain a set of $Z_{p}=Z_{p}(\beta, \Delta)$ for $p=1$ to $p=p_{\max }$. Each of these $Z_{p}$ we perturb randomly (uniformly distributed random numbers) by, at most, $\alpha \%$, which gives us a perturbed set of $\tilde{Z}_{p}$. For this set of $\tilde{Z}_{p}$ we then seek to find best-fit $\beta_{\mathrm{f}}$ and $\Delta_{\mathrm{f}}$. In the following, we do not consider one set of $\tilde{Z}_{p}$, as would be the case in a real application (unless multiple time slices are available, see Sect. 3). Instead, we take a statistical view for the problem by looking at a large number (1000 to 100000 , see below) of randomly generated sets of perturbed $\tilde{Z}_{p}$. This enables us, in a statistical sense, to relate the accuracy of the $\tilde{Z}_{p}$ with the accuracy of the fitted parameters. Our approach leaves us with two free parameters, the uncertainty $\alpha$ and the maximum order $p_{\max }$. 

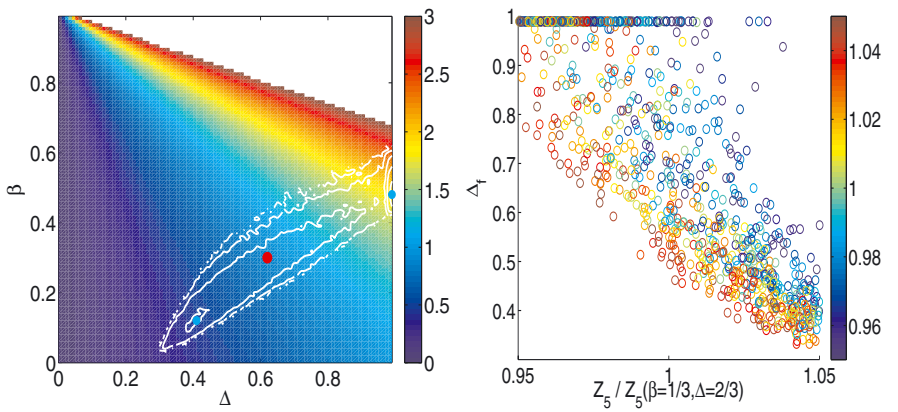

Fig. 5. Best-fit $\beta_{\mathrm{f}}$ and $\Delta_{\mathrm{f}}$ from $5 \%$ perturbed $Z_{p}$. Left: $2 \mathrm{D}$ histogram (contours, $\log 10$, spacing 0.5 , spanning three orders of magnitude) of best-fit $\beta$ - $\Delta$-values from 100000 perturbed data sets. We note that the $2 \mathrm{D}$ histogram shows two peak values (indicated by cyan dots), none of them co-located with the unperturbed $(\beta=1 / 3, \Delta=2 / 3$ ) pair (red dot). Right: underestimation of $Z_{5}$ favors $\Delta_{\mathrm{f}}=1$. Shown is, for a subset of 1000 perturbed data sets, $\Delta_{\mathrm{f}}$ as function of $\tilde{Z}_{5} / Z_{5}$, again for an unperturbed pair $(\beta=1 / 3, \Delta=2 / 3)$. Colors indicate $\tilde{Z}_{1} / Z_{1}$.

Table 1. Illustration of range $\delta C$ of co-dimension $C$ for given order $p$ and uncertainty $\delta Z_{p}$ of structure functions for two $\beta-\Delta$ pairs.

\begin{tabular}{cccc}
\hline \hline \multicolumn{4}{c}{$\beta=1 / 3, \Delta=2 / 3, C=1$} \\
\multicolumn{3}{c}{$\delta Z_{p} / Z_{p}=1 \%$} & $p=1, \ldots, 9$ \\
\hline$p=1, \ldots, 5:$ & $0.79-1.46$ & $\delta Z_{p} / Z_{p}=1 \%:$ & $0.93-1.08$ \\
$p=1, \ldots, 6:$ & $0.83-1.23$ & $\delta Z_{p} / Z_{p}=2 \%:$ & $0.84-1.27$ \\
$p=1, \ldots, 7:$ & $0.87-1.18$ & $\delta Z_{p} / Z_{p}=3 \%:$ & $0.74-1.52$ \\
$p=1, \ldots, 8:$ & $0.90-1.13$ & $\delta Z_{p} / Z_{p}=4 \%:$ & $0.70-1.92$ \\
$p=1, \ldots, 9:$ & $0.93-1.08$ & $\delta Z_{p} / Z_{p}=5 \%:$ & $0.65-2.25$ \\
\hline \multicolumn{5}{c}{$\beta=2 / 3, \Delta=2 / 3, C=2$} \\
$\delta Z_{p} / Z_{p}=1 \%$ & $p=1, \ldots, 9$ \\
\hline$p=1, \ldots, 5:$ & $0.51-3.00$ & $\delta Z_{p} / Z_{p}=1 \%:$ & $1.11-3.00$ \\
$p=1, \ldots, 6:$ & $0.70-3.00$ & $\delta Z_{p} / Z_{p}=2 \%:$ & $0.71-3.00$ \\
$p=1, \ldots, 7:$ & $0.86-3.00$ & $\delta Z_{p} / Z_{p}=3 \%:$ & $0.55-3.00$ \\
$p=1, \ldots, 8:$ & $1.00-3.00$ & $\delta Z_{p} / Z_{p}=4 \%:$ & $0.44-3.00$ \\
$p=1, \ldots, 9:$ & $1.11-3.00$ & $\delta Z_{p} / Z_{p}=5 \%:$ & $0.36-3.00$ \\
\hline
\end{tabular}

\subsubsection{Minimization of least square error in $Z_{p}$}

A straightforward way to determine best-fit $\beta_{\mathrm{f}}$ and $\Delta_{\mathrm{f}}$ for any given set of $\tilde{Z}_{p}, p=1, \ldots, p_{\max }$ is to minimize

$$
\sum_{p=1}^{p_{\max }}\left[\tilde{Z}_{p}-Z_{p}\left(\beta_{\mathrm{f}}, \Delta_{\mathrm{f}}\right)\right]^{2}
$$

over the $\beta$ - $\Delta$-plane. To find the minimum, we compare the $\tilde{Z}_{p}$ with pre-computed values $Z_{p}(\beta, \Delta)$ on a fine $\beta$ - $\Delta$-grid $(\beta, \Delta \in$ $(0,1)$; grid-spacing 0.002$)$. The associated co-dimension is given by $C_{\mathrm{f}}=\Delta_{\mathrm{f}} /\left(1-\beta_{\mathrm{f}}\right)$.

To capture the range of potential outcomes for a range of similarly perturbed data sets $\tilde{Z}_{p}$, we produced 100000 perturbed data sets, for each of which we determined $\beta_{\mathrm{f}}$ and $\Delta_{\mathrm{f}}$. For initial values $(\beta, \Delta)=(1 / 3,2 / 3)$ and (at most) $5 \%$ perturbed $Z_{p}$ for $p=1, \ldots, 5$, the result is summarized in Fig. 5 .

Shown in the left panel of Fig. 5 is a $2 \mathrm{D}$ histogram (contours) of our 100000 best-fit $\left(\beta_{\mathrm{f}}, \Delta_{\mathrm{f}}\right)$ pairs. Two points are noteworthy. First, the overall area defined by the histogram is similar to the area in Fig. 3, left panel. This is remarkable since the area in Fig. 3 is strictly defined by the $5 \%$ uncertainty of the $Z_{p}$, whereas the area in Fig. 5 is defined through a minimization problem. Second, the 2D histogram has an interior structure with two peaks, around $(\beta, \Delta)=(0.1,0.4)$ or $C=0.4$ and $(\beta, \Delta)=(0.45,1.0)$ or $C=1.8$ (cyan dots). None of them is colocated with the initial, unperturbed $(\beta, \Delta)=(1 / 3,2 / 3)$ pair (red dot, $C=1$ ).

Three questions come to mind. Where do the two peaks in the $2 \mathrm{D}$ histogram come from? Do other $(\beta, \Delta)$ pairs result in a qualitatively different picture? Can the minimization procedure be improved to better recover the initial, unperturbed $(\beta, \Delta)$ pair? We address the first two questions in the following while postponing the third question for Sect. 2.3.2.

The existence and location of the two peaks can be understood, at least qualitatively, from two observations. First, minimization via Eq. (7) gives more weight to larger $p$, as they are associated with larger values of $Z_{p}$. Roughly speaking, the bestfit $\left(\beta_{\mathrm{f}}, \Delta_{\mathrm{f}}\right)$ pair tends to lie on or close to the curve defined by $\tilde{Z}_{5}$. Moving away from that curve results in a large penalty in the form of a large contribution to the sum in Eq. (7). Second, this translates the minimization problem into the question of where the curves for $p<5$ come closest to the curve defined by $\tilde{Z}_{5}$. For illustration, we consider two extreme values of $\tilde{Z}_{5}$. To stay on the lower green curve in the left panel of Fig. $3,\left(\tilde{Z}_{5}=0.95 Z_{5}\right)$ and, at the same time, be as close as possible to any of the white curves $(p=1)$ results in a $\left(\beta_{\mathrm{f}}, \Delta_{\mathrm{f}}\right)$ pair to the right, at $\Delta_{\mathrm{f}} \approx 1$. By contrast, the upper green curve (105\% of the exact $Z_{5}$ curve) only comes closest to (intersects) any white curve between $95 \% Z_{1}$ and $105 \% Z_{1}$ in a region further to the left. Clearly, the full problem is more intricate, with also curves for $Z_{2}$ and $Z_{4}$, and the $Z_{5}$ curve not necessarily adopting one of its two extreme values. Nevertheless, Fig. 5, right panel, suggests the full data to be in line with the above reasoning. For 1000 randomly picked data sets from the left panel, we show $\Delta_{\mathrm{f}}$ as a function of $\tilde{Z}_{5} / Z_{5}$, with $Z_{5}$ the exact value. Colors indicate $\tilde{Z}_{1} / Z_{1}$. As can be seen, $\Delta_{\mathrm{f}}=1$ indeed tends to be associated with small $\tilde{Z}_{5}$ and small $\tilde{Z}_{1}$ (lower green and upper white curve in Fig. 3, left panel). Particularly low values of $\Delta_{\mathrm{f}}$ (e.g. $\Delta_{\mathrm{f}} \approx 0.4$ ) tend to occur for large $\tilde{Z}_{5}$ and any $\tilde{Z}_{1}$ (upper green curve and any white curve in Fig. 3, left panel).

Concerning other initial values (other exact $(\beta, \Delta)$ pairs), a similar situation arises in the sense that double peaked histograms emerge. Details depend, however, on the concrete values of $\beta$ and $\Delta$, on the assumed uncertainty (5\% or more/less), and on $p_{\max }$. An illustration is given in Fig. 6, by means of 1D histograms of $C_{\mathrm{f}}=\Delta_{\mathrm{f}} /\left(1-\beta_{\mathrm{f}}\right)$. These 1D histograms are less intricate than the 2D histogram in Fig. 5, left, yet still capture the essentials. We show histograms for $\Delta=2 / 3$ and different values of $\beta \in[0.0476,2 / 3]$ (corresponding to $C \in[0.7,2]$ ) and accuracies between $0.1 \%$ and $20 \%$. Five points may be made. First, the double-peaked structure that is apparent in the $\beta-\Delta$ plane in Fig. 5 re-appears as a double peak in the 1D $C_{\mathrm{f}}$-histograms of Fig. 6 (panel in row three, column three). Second, the doublepeak vanishes as $\beta$ and the uncertainty both become small (lower left corner of the figure). For the same uncertainty, the doublepeak exists for large $\beta$ but not for small $\beta$ (third row in Fig. 6). For the same $\beta$, the double-peak exists for large uncertainties but not for small ones (second column of Fig. 6). Third, going to really small values of $\beta$ and the uncertainty, the histogram becomes symmetric with one central peak. Fourth, only for these really small values is the co-dimension of the initially prescribed $(\beta, \Delta)$ pair (show in red) co-located with the peak of the histogram. Fifth, for $\beta=\Delta=2 / 3$ (right column) the histogram peaks at $C_{\mathrm{f}}=3$ instead of $C=2$, unless the accuracy is really high $(0.1 \%$, last row). This is understandable from the arguments presented above, with regards to the origin of the two peaks in the 2D histogram in Fig. 5, and from looking at the green $(p=5)$ and white $(p=1)$ curves in Fig. 4, upper left panel. 

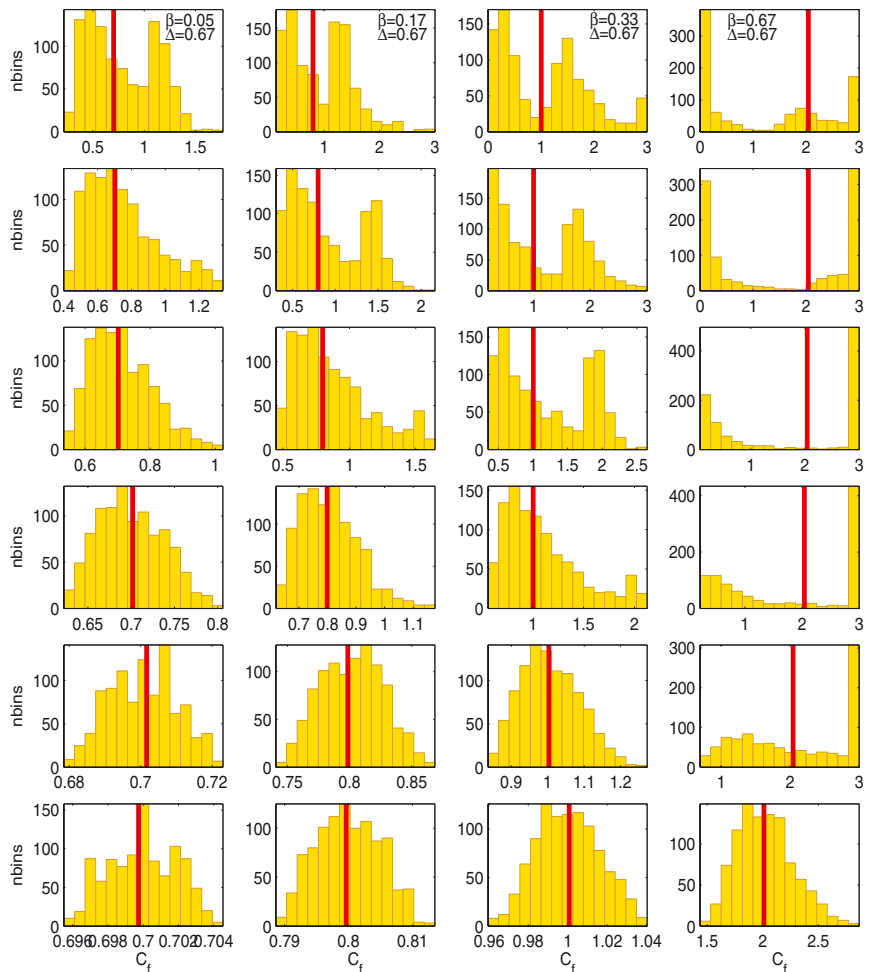

Fig. 6. Role of $\beta$ (columns) and accuracy (rows) of associated $Z_{p}$, for fixed $\Delta=2 / 3$. Shown are PDFs ( $y$-axis) of $C_{\mathrm{f}}$ ( $x$-axis), 1000 random data sets, powers $p=1, \ldots, 5$ for $\beta=0.0476$ (first column), $\beta=0.17$ (second column), $\beta=1 / 3$ (third column), and $\beta=2 / 3$ (fourth column). Corresponding exact co-dimensions (red lines) are, from left to right: $C=0.7, C=0.8, C=1$, and $C=2$. Individual rows from top to bottom contain accuracies of $20 \%, 10 \%, 5 \%, 2 \%, 0.5 \%$, and $0.1 \%$. As can be seen, the larger $\beta$, the more severe are the consequences of inaccuracies in the $Z_{p}$. Histograms in the upper right (large $\beta$, low accuracy of the $Z_{p}$ ) look worst. We note that axis ranges differ among panels, to best capture the shape of each histogram.

In summary, unless both, $\delta Z_{p}$ and $\beta$ are small, best-fit values will preferentially reside in either one of the two peaks of the histograms in Figs. 5 or 6 instead of merely scattering around the correct solution, as in Fig. 6, lower left panel.

\subsubsection{Alternative ways to obtain best-fit $\beta_{\mathrm{f}}$ and $\Delta_{\mathrm{f}}$}

A number of ideas come to mind on how one may improve the best-fit approach detailed in Sect. 2.3.1.

Recalling the findings in Sect. 2.2.1, including higher values of $p$ in the best-fit estimate should improve the situation. From Fig. 7 it can be taken that this is indeed the case, at least for the example shown $(\beta=1 / 3, \Delta=2 / 3$, accuracy of $5 \%)$. However, the improvement may be regarded as rather modest. Going from $p=5$ to $p=10$, as is illustrated in the figure, has about the same effect as staying with $p=5$ but going from an accuracy of $5 \%$ to an accuracy of $2 \%$. From a practical point of view it also seems questionable whether high order structure functions can meet the accuracy requirements. In numerical simulations, higher order structure functions are probably more prone to the bottleneck effect (Dobler et al. 2003; Kritsuk et al. 2007a).

Another way to improve the situation could be to go to weighted root mean squares instead of the unweighted sum in Eq. (7). Hopefully this breaks the dominant role of the highest order $Z_{p}$ available (see Sect. 2.3.1) and, ultimately, leads to more
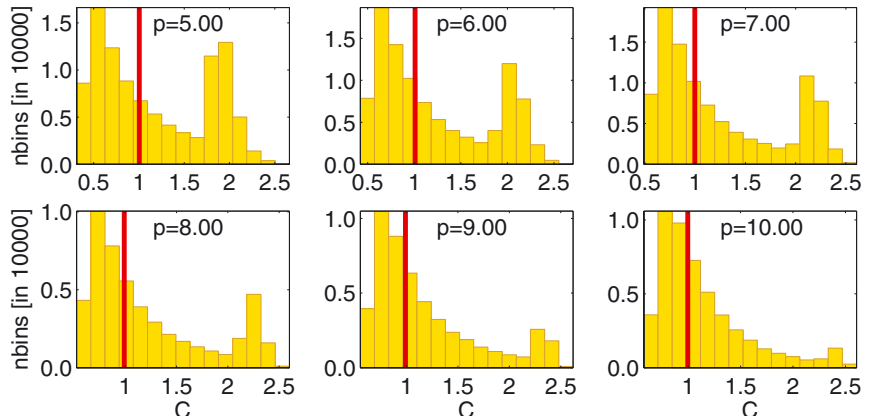

Fig. 7. Role of higher order moments. Inclusion of higher order ESS scaling exponents (from $p=5$, top left, to $p=10$, bottom right) gradually reduces the erroneous peak in the best-fit co-dimension (around $C_{\mathrm{f}}=2.5$ ). Shown are PDFs of $C_{\mathrm{f}}$ for at most $5 \%$ perturbed $Z_{p}$ values (100000 random data sets) and exact pair $(\beta=1 / 3, \Delta=2 / 3)$. Spacing of the $\beta$ - $\Delta$-grid for fitting is 0.002 .

accurate best-fit $\beta_{\mathrm{f}}$ and $\Delta_{\mathrm{f}}$. Two weightings come to mind. On the one hand, weights proportional to the inverse of the $Z_{p}$ with the goal of giving equal weight to each term in the sum, thus reducing the "overweight" of larger $p$ in the sum. On the other hand, we could try to give more weight to $p$ terms with a higher accuracy (smaller $\delta Z_{p}$ ). Corresponding information may be available, e.g. from the numerical determination of the $Z_{p}$. We tried both ideas but neither choice of weights decidedly improved the bestfit values. Weighting tends to change the relative height of the two peaks in the double peaked histograms of Fig. 6, but it does not get rid of the double peaked structure.

We interpret this finding in the following way. First, there are likely always several $Z_{p}$ that do not have their exact values and thus draw the solution in different directions, away from its exact value. Second, the different curve shapes are important so that, even for weighted sums, the terms $p=1$ and $p=p_{\max }$ are of crucial importance for the overall fit.

In summary, none of the above alternative ways of fitting simultaneously for $\beta$ and $\Delta$ provides clearly superior results to what can be obtained from the straightforward minimization of Eq. (7). We conclude that, for successful two-parameter fits of $\beta$ and $\Delta$, highly accurate $Z_{p}$ are a must. A quantitative estimate of "highly accurate"is given in the next section.

\subsubsection{Required accuracy of $Z_{p}$ for "good" best-fit $\beta_{f}$ and $\Delta_{f}$}

We now ask how accurate the $Z_{p}$ have to be in order to reach a prescribed accuracy of $C_{\mathrm{f}}=\Delta_{\mathrm{f}} /\left(1-\beta_{\mathrm{f}}\right)$ via fitting $\beta_{\mathrm{f}}$ and $\Delta_{\mathrm{f}}$.

We formulate our accuracy goal in terms of only $C_{\mathrm{f}}$, since we illustrated in Sect. 2.3.1 that a single peaked and roughly symmetric distribution of $C_{\mathrm{f}}$ goes hand in hand with high accuracy, not only of $C_{\mathrm{f}}$ but also of the underlying two parameter fit, $\beta_{\mathrm{f}}$ and $\Delta_{\mathrm{f}}$. If the latter is not accurate enough, a double peaked distribution for $C_{\mathrm{f}}$ results. We find, as a rule of thumb, a single peak distribution if $2 / 3$ of all $C_{\mathrm{f}}$ lie within $10 \%$ or better of the exact $C$. We use Eq. (7) for the two parameter fit, as the more elaborate attempts of Sect. 2.3.2 gave no decidedly better results. As theoretical arguments suggest $\Delta=2 / 3$ or larger (Dubrulle 1994; Schmidt et al. 2008), we concentrate on that part of the $\beta$ - $\Delta$-plane.

In practical terms, we define a grid of exact pairs $(\beta, \Delta)$ via a (nearly) equidistant grid of $C=0.4, \ldots, 2$ and $\Delta=$ $0.7, \ldots, 0.99$ plus, in addition, $\Delta=2 / 3$. We equally define some fixed levels of perturbations: $\delta Z_{p} / Z_{p}$ (in $\%$ ) $\in$ $[0.05,0.1,0.2,0.5,1,2,5,10,15,20]$. For each exact pair and 


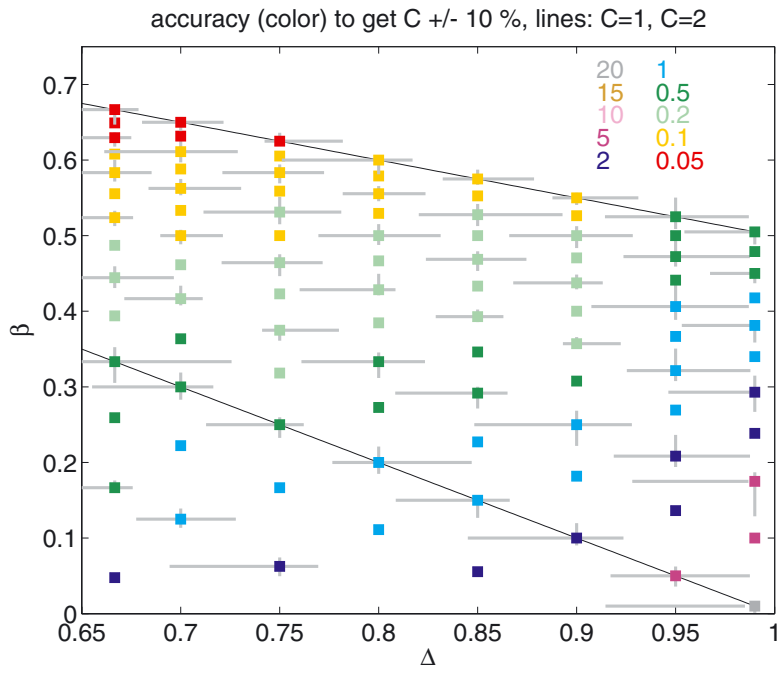

accuracy (color) to get $\mathrm{C}+/-5 \%$, lines: $\mathrm{C}=1, \mathrm{C}=2$

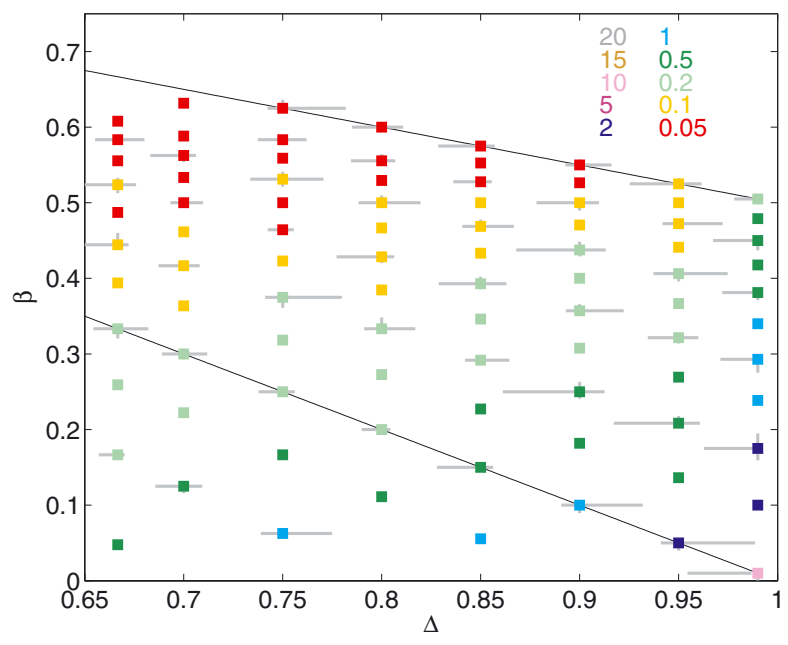

Fig. 8. Required accuracy (color coding) of perturbed $Z_{p}, p=1, \ldots, 5$, such that for at least $2 / 3$ of the best-fit pairs $\left(\beta_{\mathrm{f}}, \Delta_{\mathrm{f}}\right)$ the associated $C_{\mathrm{f}}$ are within $10 \%$ (top) or 5\% (bottom) of the $C$ associated with the initial, unperturbed $(\beta, \Delta)$ pair. As can be seen, the required accuracy depends crucially on the location within the $\beta-\Delta$ plane. Gray lines indicate the range within which $2 / 3$ of the actual $\beta_{\mathrm{f}}$ (vertical direction) and $\Delta_{\mathrm{f}}$ (horizontal direction) lie. Black lines indicate constant $C=1$ (lower line) and $C=2$ (upper line). For details see text.

each perturbation, we created 1000 perturbed sets of $\tilde{Z}_{p}, p=$ $1, \ldots, 5$ (see Sect. 2.3). Each perturbed set is fitted via Eq. (7). For each initial pair $(\beta, \Delta)$ and for each prescribed $\delta Z_{p} / Z_{p}$ this yields 1000 fitted pairs $\left(\beta_{\mathrm{f}}, \Delta_{\mathrm{f}}\right)$ and derived co-dimensions $C_{\mathrm{f}}$. These can, in principle, be arranged in histograms, as in Fig. 6. Finally, we identify the largest $\delta Z_{p} / Z_{p}$ for which $2 / 3$ of all $C_{\mathrm{f}}$ lie within the demanded accuracy of the exact, initial $C$.

Figure 8 illustrates the result. Obviously, the accuracy of the $\tilde{Z}_{p}$ (colored squares, in \%) that is needed to get at least $2 / 3$ of best-fit $C_{\mathrm{f}}$ to lie within $10 \%$ (top panel) or 5\% (bottom panel) of the exact (initial) $C$ depends on the position within the $\beta-\Delta$ plane. In the lower right parts, $\delta Z_{p} / Z_{p} \geq 1 \%$ is sufficient to get $10 \%$ accurate $C_{\mathrm{f}}$, and $\delta Z_{p} / Z_{p} \geq 0.5 \%$ yields $5 \%$ accurate $C_{\mathrm{f}}$. By contrast, in the upper left parts of the plane $(\beta \geq 0.5)$ one needs $\delta Z_{p} / Z_{p} \leq 0.1 \%$ to get $10 \%$ accurate $C_{\mathrm{f}}$. Gray lines in Fig. 8 (for clarity only shown for a subset of the colored squares) indicate the range within which at least $2 / 3$ of the actually fitted $\beta_{\mathrm{f}}$ and $\Delta_{\mathrm{f}}$ lie. The range is larger for $\Delta$ (horizontal lines) than for $\beta$ (vertical lines). This is plausible from Fig. 4 , from the area confined by multiple $Z_{p}$ curves. Repeating Fig. 8 but demanding $10 \%$ accuracy for $\Delta_{\mathrm{f}}$ instead of $C_{\mathrm{f}}$ yields a similar pattern in the $\beta$ - $\Delta$-plane (not shown), while demanding $10 \%$ accuracy for $\beta_{\mathrm{f}}$ gives a much more homogeneous pattern $\left(0.2 \%\right.$ to $0.5 \%$ accuracy for $\tilde{Z}_{p}$, not shown).

For highly compressible turbulence, best-fit $\beta_{\mathrm{f}}$ and $\Delta_{\mathrm{f}}$ are expected to lie in the lower part of the $\beta$ - $\Delta$-plane in Fig. 8 , roughly $\beta \leq 1 / 3$ and $\Delta \geq 2 / 3$ (Boldyrev 2002; Padoan et al. 2004; Pan et al. 2009). Here, accuracies of $0.5 \%, 0.1 \%$, and $0.05 \%$ for the $\tilde{Z}_{p}$ translate into accuracies of $10 \%, 2 \%$, and $1 \%$ for $\beta_{\mathrm{f}}$ and $\Delta_{\mathrm{f}}$ (not shown). Fits of similar quality require much more accurate $\tilde{Z}_{p}$ in the mildly compressible regime, where $\beta>1 / 3$ (upper part of panels in Fig. 8) and ultimately $\beta=2 / 3$ in the incompressible limit. We note that in practical applications, best-fit values may be further improved by combining, for example, data from different time slices (Pan et al. 2009).

In summary, a $2 \%(1 \%)$ accurate simultaneous fit for $\beta_{\mathrm{f}}$ and $\Delta_{\mathrm{f}}$ should be possible in the highly compressible regime if the $Z_{p}$ are $0.1 \%(0.05 \%)$ accurate. If no satisfying fit is possible for such $Z_{p}$, this may indicate that the model is not applicable to the turbulence data under examination.

\section{Discussion}

We address three topics. First, can the necessary accuracy for the $Z_{p}$ be met in practical applications? Second, if we had this type of accurate simulation data, what could be learned about the hierarchical structure model and its applicability or non-applicability to driven, isothermal, supersonic turbulence in a $3 \mathrm{D}$ periodic box? Third, we want to briefly revisit the frequently used one parameter fits.

We start with the question whether $0.1 \%$ or even $0.05 \%$ accurate $Z_{p}$ for $p=1, \ldots, 5$ are achievable, as are needed to get $2 \%(1 \%)$ accurate fits for $\beta$ and $\Delta$. The answer is probably yes, at least in the context of $3 \mathrm{D}$ periodic box simulations. Schmidt et al. (2008) estimate the accuracy of their $Z_{p}, p=1, \ldots, 5$, to $1 \%$ (3D box simulations, $1024^{3}$ ). Kritsuk et al. (2007a) estimate $1 \%$ accuracy or better for absolute scaling exponents $\zeta_{p}, p=1, \ldots, 3$ (3D box simulations, $1024^{3}$ ). Meanwhile, 3D box simulations with $4096^{3}$ exist (e.g. Federrath 2013; Beresnyak 2014). A first order estimate suggests the four times better resolution to translate into four times (first order scheme) or 16 times (second order scheme) more accurate structure functions, thus accuracies of $0.25 \%$ or even $0.0625 \%$. Moreover, if the accuracy of the $Z_{p}$ is good enough to avoid double peaked histograms as in Fig. 6, accuracy may be further enhanced by exploring multiple time slices. Pan et al. (2009; 3D box simulations, $\left.1024^{3}\right)$ used data from nine time slices for their two parameter fit. Their work is comparable although they rely on dissipation rates instead of velocity structure functions, since the involved scaling exponents ( $\tau_{p}$ for the dissipation rate) are structurally similar according to Kolmogorov's refined similarity hypothesis (Kolmogorov 1962), $\zeta_{p}=p / 3+\tau_{p / 3}$. The tests we carried out using ratios of $\tau_{p}$ instead of $\zeta_{p}$ indeed show a similar behavior. From the quality of their fit and based on our results, we estimate their $\tau_{p}$ to be about $1 \%$ accurate, which is plausible given their numerical resolution. A reliable two parameter fit to $3 \mathrm{D}$ periodic box data of highly supersonic turbulence that is based on velocity structure functions thus appears feasible with today's data.

What could be learned from better simulation data $\left(4096^{3}\right.$ or better) and associated, more accurate $Z_{\mathrm{p}}$ ? Each $Z_{\mathrm{p}}$ defines a curve with associated uncertainty in the $\beta-\Delta$ plane, the curves 
for different $p$ may or may not intersect to within uncertainties. If they intersect, the model by She \& Leveque (1994) may indeed carry over to highly compressible turbulence. It is then interesting to see whether the fitted range for $\Delta$, currently estimated as $(0.67-0.78)$ by Pan et al. (2009), remains compatible with $\Delta=2 / 3$, the value tacitly assumed in a large body of literature. It is also interesting to check whether $\beta=1 / 3$, as theoretically anticipated by Boldyrev (2002). If indeed $(\beta, \Delta)=(1 / 3,2 / 3)$, results from one parameter fits that quantify the transition to incompressible turbulence $(\beta=\Delta=2 / 3)$ with decreasing Mach number likely apply (Padoan et al. 2004). If $\Delta \neq 2 / 3$, two parameter fits for $\beta$ and $\Delta$ would also be needed in the mildly compressible regime. However, the analysis in Sect. 2.3.3 suggests that these kind of fits are likely beyond reach of today's computer resources.

The latter of the above cases, where the $Z_{p}$ curves do not intersect to within their uncertainty, would imply that the model by She \& Leveque (1994) does not carry over to 3D periodic box simulations of driven, isothermal, highly compressible turbulence. A simple reason here could be that theoretical results are based on the assumption of an infinite Reynolds number, a criterion clearly violated by numerical simulations. More importantly, She \& Waymire (1995) already pointed out that there is no reason why only one dimension should be associated with the most dissipative structure. They argued that in such a large portion of space as is typically analyzed, a variety of most dissipative structures may co-exist with different codimensions. Hopkins (2013) suggests a slightly different model based on work by Castaing (1996), which is more compatible with not strictly log-normal density PDFs as observed in isothermal supersonic turbulence. Finally, yet other models exist, (e.g. via multifractals Macek et al. 2011; Zybin \& Sirota 2013), as well as other perspectives on the fractal character of a turbulent medium (see e.g. Kritsuk et al. 2007a).

Lastly, we briefly come back to the one parameter fits that are often used in the literature. Fixing the value of $\Delta$ by hand greatly reduces the impact of uncertainties in the $Z_{p}$ on the accuracy of the estimated best-fit co-dimension $C_{\mathrm{f}}$. Folini et al. (2014) found $5 \%$ uncertain $Z_{p}, p=1, \ldots, 5$, to translate into roughly $10 \%$ uncertainty of the $C_{\mathrm{f}}$ for fixed $\Delta=2 / 3$. Fig. 3 offers a qualitative understanding of this reduced "error propagation", which suggests some sensitivity to the specific location in the $\beta$ - $\Delta$-plane, and indicates that fixing $C$ or $\beta$ instead of $\Delta$ has a similar effect. Fixing $\beta$ seems questionable at first sight since there is, to our knowledge, little theoretical understanding of what numerical value $\beta$ might have (see e.g. Dubrulle 1994). On the other hand, She et al. (2001) presented a theoretical framework that allows for an independent determination of only $\beta$ from the relative scaling exponents $Z_{p}$ (see also Hily-Blant et al. (2008), their Appendix A3). One could thus imagine breaking the two parameter fit for $\beta$ and $\Delta$ into a two step procedure: first, fix the value of $\beta$, then use this value and do a one parameter fit for $\Delta$. Hopefully this type of a two step approach is more robust against uncertainties in the $Z_{p}$, but this question is beyond the scope of the current paper and we are unaware of corresponding attempts in the literature.

\section{Summary and conclusions}

This study was motivated by the overarching question of whether or not the random cascade model (She \& Leveque 1994; Dubrulle 1994; She \& Waymire 1995; Boldyrev 2002) applies to simulation data of highly compressible isothermal turbulence and, if so, with what parameter values for $\beta$ and $\Delta$. If applicable, the model offers a theoretical link between observable properties of the turbulence, namely ratios $Z_{p}$ of scaling exponents of the structure functions, and non-observable turbulence characteristics, for example the dimension $D$ of the most dissipative structures. To date, applicability of the model is assumed in much of the literature with $\Delta=2 / 3$, a value just marginally compatible with simulation-based best estimates (Pan et al. 2009): $\Delta=0.71$ with an uncertainty range $\Delta \in(0.67,0.78)$.

We examine how uncertainties in the $Z_{p}$ translate into uncertainties of best-fit $\beta$ - $\Delta$-pairs and discuss what best-fits, consequently, seem achievable with today's computer resources. A Monte Carlo approach is used to mimic actual simulation data. The results can be summarized in six main points.

- Simultaneous fitting of $\beta$ and $\Delta$ to sets of substantially (5\%) perturbed (uncertain) $Z_{p}$ yields a "double peaked ridge" of best-fit values in the $\beta-\Delta$ plane. None of the two peaks is co-located with the initial $(\beta, \Delta)$ pair.

- The highest and lowest order $p$ are particularly relevant for simultaneous fitting of $\beta$ and $\Delta$. A somewhat optimal choice is $p=1, \ldots, 5$. Yet higher order structure functions add comparatively little to the quality of the fit, while they tend to be afflicted with larger uncertainties in real applications.

- A simultaneous, $2 \%$ (1\%) accurate fit of $\beta$ and $\Delta$ should be possible if the $Z_{p}, p=1, \ldots, 5$, are $0.1 \%(0.05 \%)$ accurate and if the (yet to be determined) value of $\beta$ is about $1 / 3$ or less.

- Applicability of the model thus may be best tested in the highly compressible regime, where $\beta \approx 1 / 3$ is expected, and not in the mildly compressible regime where $\beta$ ultimately must approach its incompressible value of $2 / 3$.

- We argue that today's computer resources likely allow to reach this accuracy. Existing simulations of $4096^{3}$ (Federrath 2013; Beresnyak 2014) probably allow for at least $2 \%$, possibly $1 \%$ accurate estimates of $\beta$ and $\Delta$.

- Should the ambiguity in the determination of $\beta$ and $\Delta$ persist despite such highly accurate $Z_{p}$, this may indicate that the notion of She $\&$ Waymire $(1995 ; \beta$ and $\Delta$ take a continuum of values) or Hopkins (2013; a different model for the statistics of the inertial range) is correct or that yet a different turbulence model is needed in this regime.

While the authors lack the computational resources to really test the estimates presented here, this study may encourage other groups to analyze their data in the light of this study.

Acknowledgements. R.W. and D.F. acknowledge support from the French National Program for High Energies PNHE. We acknowledge support from the Pôle Scientifique de Modélisation Numérique (PSMN), from the Grand Equipement National de Calcul Intensif (GENCI), project number x2014046960, and from the European Research Council through grant ERCAdG No. 320478-TOFU.

\section{References}

Benzi, R., Ciliberto, S., Tripiccione, R., et al. 1993, Phys. Rev. E, 48, 29 Beresnyak, A. 2014, ApJ, 784, L20

Boldyrev, S. 2002, ApJ, 569, 841

Boldyrev, S., Nordlund, A., \& Padoan, P. 2002, Phys. Rev. Lett., 89, 031102

Castaing, B. 1996, J. Phys. II, 6, 105

Chabrier, G., \& Hennebelle, P. 2011, A\&A, 534, A106

Dobler, W., Haugen, N. E., Yousef, T. A., \& Brandenburg, A. 2003, Phys. Rev. E, 68, 026304

Dubrulle, B. 1994, Phys. Rev. Lett., 73, 959 
A\&A 587, A120 (2016)

Federrath, C. 2013, MNRAS, 436, 1245

Federrath, C., \& Klessen, R. S. 2012, ApJ, 761, 156

Folini, D., Walder, R., \& Favre, J. M. 2014, A\&A, 562, A112

Gustafsson, M., Brandenburg, A., Lemaire, J. L., \& Field, D. 2006, A\&A, 454, 815

Hennebelle, P., \& Chabrier, G. 2008, ApJ, 684, 395

Hily-Blant, P., Falgarone, E., \& Pety, J. 2008, A\&A, 481, 367

Hobbs, A., Nayakshin, S., Power, C., \& King, A. 2011, MNRAS, 413, 2633

Hopkins, P. F. 2013, MNRAS, 430, 1880

Kolmogorov, A. N. 1962, J. Fluid Mech., 13, 82

Kritsuk, A. G., Norman, M. L., Padoan, P., \& Wagner, R. 2007a, ApJ, 665, 416

Kritsuk, A. G., Padoan, P., Wagner, R., \& Norman, M. L. 2007b, in Turbulence and Nonlinear Processes in Astrophysical Plasmas, eds. D. Shaikh, \& G. P. Zank, AIP Conf. Ser., 932, 393

Kritsuk, A. G., Lee, C. T., \& Norman, M. L. 2013, MNRAS, 436, 3247

Lazar, A., Nakar, E., \& Piran, T. 2009, ApJ, 695, L10

Le Maître, O. P., \& Knio, O. M. 2010, Spectral Methods for Uncertainty Quantification (Springer)
Macek, W. M., Wawrzaszek, A., \& Carbone, V. 2011, Geophys. Res. Lett., 38, 19103

Najm, H. N. 2009, Ann. Rev. Fluid Mech., 41, 35

Narayan, R., \& Kumar, P. 2009, MNRAS, 394, L117

Padoan, P., Jimenez, R., Nordlund, Å., \& Boldyrev, S. 2004, Phys. Rev. Lett., 92, 191102

Padoan, P., Haugbølle, T., \& Nordlund, Å. 2012, ApJ, 759, L27

Pan, L., Padoan, P., \& Kritsuk, A. G. 2009, Phys. Rev. Lett., 102, 034501

Schmidt, W., Federrath, C., \& Klessen, R. 2008, Phys. Rev. Lett., 101, 194505

Schmidt, W., Federrath, C., Hupp, M., Kern, S., \& Niemeyer, J. C. 2009, A\&A, 494, 127

She, Z.-S., \& Leveque, E. 1994, Phys. Rev. Lett., 72, 336

She, Z.-S., \& Waymire, E. C. 1995, Phys. Rev. Lett., 74, 262

She, Z.-S., \& Zhang, Z.-X. 2009, Acta Mech. Sin., 25, 279

She, Z.-S., Ren, K., Lewis, G. S., \& Swinney, H. L. 2001, Phys. Rev. E, 64, 016308

Walder, R., Folini, D., \& Shore, S. N. 2008, A\&A, 484, L9

Zybin, K. P., \& Sirota, V. A. 2013, Phys. Rev. E, 88, 043017 